\title{
Findings at Left Heart Cardiac Catheterization of Nonagenarians
}

\author{
By C. Richard Conti, MD, MACC, Editor-in-Chief
}

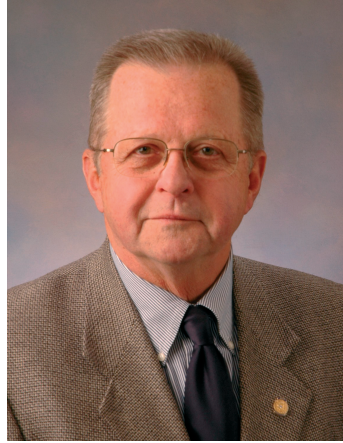

It is estimated that the average 80 -year-old person will live an additional eight years and 40\% will have symptomatic cardiovascular disease. Few studies exist in patients 90 years or older. Several years ago, a prominent pathologist made the point that at autopsy, nonagenarians rarely had significant coronary disease. That does not seem to be the case in 2009 .

\section{Background}

Those living past 90 years may be doing so because of aggressive strategies combining noninterventional therapy with interventional procedures. There are some data relating to cardiac surgery in nonagenarians, but these papers do not report the complications related to cardiac catheterization prior to surgery. Recently, I had a patient, age 91 , who had refractory angina and thus had a clinical indication for coronary angiography to determine whether or not revascularization could be performed. This patient's case stimulated me to evaluate our experience in this select group of patients.

\section{Methods}

A retrospective review of cardiac catheterization data in nonagenarians at the University of Florida since the year 2000 .

\section{Population}

Fourteen nonagenarians who underwent cardiac catheterization between the years 2000 and 2008. Eight were male, and six were female. Average age was 92.2 years with a range of 90 to 95 years. Six of those patients were age 92 .

Diagnostic coronary angiography was performed in all 14 patients; percutaneous coronary intervention $(\mathrm{PCI})$ was performed in 8 of the 14 . The indications for cardiac catheterization were: acute coronary syndromes (3); valvular disease and heart failure (1); chronic ischemic heart disease (7); ST segment elevation myocardial infarction (2); and assessment prior to permanent pacing (1).

\section{Results}

The coronary artery findings were as follows: All patients had angiographic evidence for multivessel coronary artery disease, although not all had high-grade stenosis. Eight (8) had some disease of the coronary artery, 13 had left anterior descending disease, 12 had circumflex disease, and 11 had right coronary artery disease.

\section{Complications}

Ten (10) patients had no complications during cardiac catheterization. There were two major complications related to cardiac catheterization: 1) Coronary perforation resulting in coronary bypass graft and eventual death, and 2) One patient fibrillated during PCI but was resuscitated successfully.

Three patients died but not necessarily related to cardiac catheterization: One presented with cardiogenic shock which despite use of intraaortic balloon counterpulsation, eventually led to death. One patient presented with ST elevation $\mathrm{MI}$ and underwent $\mathrm{PCI} /$ stent. One patient died early after triple valve surgery, following an uncomplicated cardiac catheterization.

\section{Conclusions}

In this cohort of 14 patients over the age of 90 , the majority of patients who underwent "clinically indicated" left heart catheterization had coronary artery disease that was generally multivessel and was clinically significant. The major complication rate for performing left heart catheterization and coronary angiography in these very elderly patients was higher (14.2\%) than the usual patient undergoing these procedures and led to death following valve surgery in one patient undergoing multivalve replacement.

When evaluating these very elderly patients for potential interventional procedures, one must remember that the risk for cardiac catheterization is higher than in the younger population, and the odds of patients over 90 years of age living many more years is quite small.

Thus, the major indication for assessment for possible intervention in nonagenarians is to relieve or stabilize symptoms and improve quality, not quantity, of life.

\section{References}

1. US Bureau of the Census, Washington, DC. Projections of the population of the United States by age, sex, and race: 1983-2008. Current Population Report 1984.

2. Edwards M-B, Taylor KM. Outcomes in Nonagenarians after heart valve replacement operation. Ann Thorac Surg. 2003;75: $830-834$. 